\title{
ENDOR SPECTRUM AND WAVEFUNCTION FOR THE RELAXED-EXCITED STATE OF THE F CENTER IN KBr
}

\author{
G. BALDACCHINI \\ Laboratori Nazionali di Frascati del CNEN, Frascati, Italy \\ and L. F. MOLLENAUER
}

Bell Laboratories, Holmdel, New Jersey, USA

\begin{abstract}
Résumé. - La densité de probabilité radiale de l'Etat Excité Relaxé (RES) du centre F dans $\mathrm{KBr}$ a été déduite de mesures de fréquences hyperfines.

Les résultats ne permettent pas de choisir entre les états $|2 s\rangle$ et $|2 p\rangle$. Cependant, la densité de probabilité expérimentale peut être assez bien représentee par un état diffus $\mid 2 \mathrm{p}>$ omettant la dépendance angulaire.

Abstract. - The radial probability density of the Relaxed Excited State (RES) of the F center in $\mathrm{KBr}$ has been extracted from measured hyperfine frequencies.

The results do not allow statements to be made about the model of $|2 \mathrm{~s}\rangle$ versus $\mid 2 p>$ state However the experimental probability density can be represented fairly well by a difiuse $|2 \mathrm{p}\rangle$ state lacking the angular dependence.
\end{abstract}

1. General considerations and previous results. Recently L. F. Mollenaluer and coworkers have been able to detect for the first time the ENDOR resonance in the relaxed-excited state (RES) of the $F$ center in $\mathrm{KI}$ [1], [2]. The most difficult and fundamental problems result from the short lifetime $\tau$ of the RES. Indeed in order to affect the F center optical cycle [3] the electron has to "feel» the nuclear reversal during its stay in the RES. Hence the nuclear direction must be reversed in a time shorter than $\tau$. Owing to the fact that the linewidth of the nuclear resonances is pratically determined by the short lifetime $\tau$, the last condition becomes

$$
(I+m)(I-m+1)\left(i_{n} H_{1} \tau\right)^{2} \geqslant 1
$$

where $\gamma_{n}$ is the gyromagnetic ratio of the nuclei and $H_{1}$ is one half the amplitude of the oscillating rf tield, $H_{\mathrm{rf}}=2 H_{1}$. We have used in (1) the normal transition probability from the state $m$ to $m-1$ for a system of nuclear spins with angular momentum $I$. We disregarded completely the enhancement of the nuclear transition probability [4] which occurs in the presence of an hyperfine interaction. Hence the condition (1) is more restrictive than the true one. Keeping this in mind we will use the sign equal in (1) from now on.

In general a field $H_{\mathrm{r}}$ of the order of $100 \mathrm{Oe}$ and at a frequency of several tens of $\mathrm{MHz}$ is necessary 10 satisfy the (1) in a typical ENDOR experiment. The problem of generating such high $H_{\mathrm{rf}}$ was solved by Mollenauer et al. [2] by using an ingenious experi- mental arrangement. We will not describe here the apparatus, which was done elsewere, but we will be content to say that the rf field is generated by two loops of copper wire, $\varnothing \simeq 0.2 \mathrm{~mm}$, which enclose an area of $\sim 1 \mathrm{~mm}^{2}$. From (1) we calculate $H_{\mathrm{rf}}=50 \mathrm{Oe}$ for $\mathrm{F}$ center in $\mathrm{Kl}, \tau=3.2 \times 10^{-6} \mathrm{~s}$ [5]. This implies at $30 \mathrm{MHz}$ and at the liquid helium temperature a power dissipation by the loop of $8 \mathrm{~mW}$. Now because of the Kapitza resistence [6] the temperature of the copper wires is $\sim 0.7 \mathrm{~K}$ higher than the bath temperature, $T_{0}=1.7 \mathrm{~K}$. It is clear that in such conditions the system is highly unstable. Much worse is the situation for $\mathrm{F}$ center in $\mathrm{KBr}$ where $\tau=2 \times 10^{-6} \mathrm{~s}$ [7]. In this case $H_{\mathrm{rf}}=100$ Oe and the power dissipation is $30 \mathrm{~mW}$, which brings the temperature of the loop $\sim 2.5 \mathrm{~K}$ higher than the bath. This means that if we use the maximum experimentally acceptable $H_{\mathrm{rr}}$ for both $\mathrm{KI}$ and $\mathrm{KBr}$ crystals, the signal to noise ratio is much worse in $\mathrm{KBr}$ than in $\mathrm{KI}$.

The ENDOR spectrum of $F$ center in $\mathrm{KI}$ revealed with this apparatus is fairly good and the hyperfine frequencies are so well resolved that we were able to extract from them the radial probability density for the RES [8]. In the case of $\mathrm{KBr}$ another difficulty has to be considered. The ENDOR spectrum contains two spectra due to the isotopes $\mathrm{Br}^{79}\left(I=\frac{3}{2}\right.$, $g \mu_{\beta} / h=1066.7 \mathrm{~Hz} / \mathrm{G}, \sim 50 \%$ natural abundance) and $\mathrm{Br}^{81}\left(I=2_{2}^{3}, g / \mu_{f} / h=1149.9 \mathrm{~Hz} / \mathrm{G}, \quad \sim 50 \%\right.$ natural abundance); $g$ is the nuclear spectroscopic splitting factor and $\mu_{\beta}$ is the Bohr magneton. Nevertheless we succeeded in detecting the ENDOR spec- 
trum in $\mathrm{KBr}$ [8]. As expected the spectrum was not resolved and the identification of the hyperfine frequencies was almost impossible. Only the maximum hyperfine frequency was extracted from it, i. e.

$$
\left(v_{\mathrm{hf}}\right)_{\max } \simeq 11.8 \mathrm{MHz} \text {. }
$$

If $P\left(\rho_{i}\right)$ is the electron probability density at the nuclear site $i$, where $\rho$ is expressed in units of the nearest-neighbor distance $d=3.298 \AA$ for $\mathrm{KBr}$, the hyperfine frequency is given by :

$$
\left(v_{\mathrm{hr}}\right)_{i}=\operatorname{CAP}\left(\rho_{i}\right)
$$

where $A$ is the amplification factor and $C$ is $2.3192 \mathrm{MHz}$ for $\mathrm{Br}^{79}$ and $2.500 \mathrm{I}$ for $\mathrm{Br}^{81}$. Assuming as for $\mathrm{F}$ center in $\mathrm{KI}$ that the ESR linewidth is due to hyperfine broadening, we have :

$$
\Delta H=2.020\left\{0.5\left[\sum_{i}\left(v_{\mathrm{hf}}\right)_{i}^{2}\right]^{1 / 2}+0.5\left[\sum_{j}\left(v_{\mathrm{hf}}\right)_{j}^{2}\right]^{1 / 2}\right\}
$$

where $\Delta H$ is the full width at half power points of the ESR line of the RES and the sums over $i$ and $j$ refer to the isotopes $\mathrm{Br}^{79}$ and $\mathrm{Br}^{81}$ respectively. Since the nuclear Zeeman frequencies at the magnetic field used are $v_{\mathrm{Z}}=21.52 \mathrm{MHz}\left(\mathrm{Br}^{79}\right)$ and $v_{\mathrm{Z}}=23.20 \mathrm{MHz}$ $\left(\mathrm{Br}^{81}\right)$ and the ENDOR frequencies are given by $v_{\mathrm{E}}=v_{\mathrm{Z}}+v_{\mathrm{hf}}$, the maximum hyperfine frequency measured refers to the $\mathrm{Br}^{81}$ isotope. Assuming as for the RES of $F$ center in KI a pure $\mid 2 p>$ state without angular dependence :

$$
P(\rho)=\left(\frac{\eta^{3}}{3 \pi}\right)(\eta \rho)^{2} \exp (-2 \eta \rho)
$$

and taking in account the experimental value of the ESR linewidth $\Delta H=270 \mathrm{Oe}$, we obtain, from (2) and (3), $\eta \simeq 0.48$ and $A \simeq 3000$ for the RES of $F$ center in $\mathrm{KBr}$.

2. Actual results. - The method followed in the previous section to calculate the values of $\eta$ and $A$ is highly unsatisfactory. First of all we used only one feature of the ENDOR spectrum, i. e. $\left(v_{h f}\right)_{\max }$, without matching the electron probability density $P(\rho)$ to the whole spectrum. Secondly, the experimental value of $\left(v_{\mathrm{hf}}\right)_{\max }$ is approximated within $\sim 10 \%$ which reflects on $\eta$ and $A$.

For these reasons we tried to extract more information from the experimental ENDOR spectra. Indeed we obtained several spectra relalive to different samples, always hoping to get a good one, which was never the case. Later we analysed these spectra more carefully taking the mean value of 12 (twelve) of them point by point. In this manner the $S / N$ ratio is expected to improve by a factor $\sim \sqrt{12}=3.5$. In figure $1 a$, solid curve, the ENDOR spectrum so

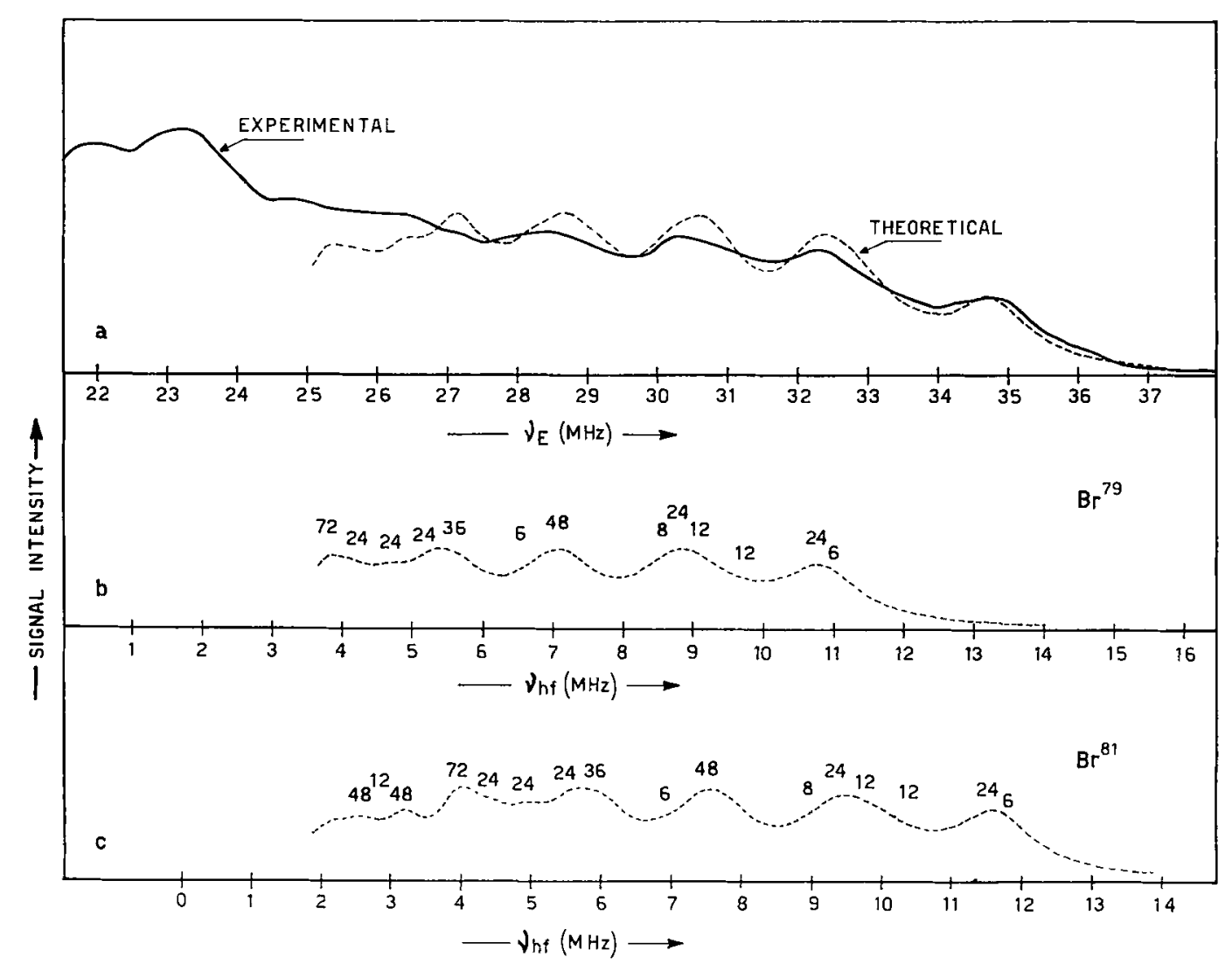

FIG. 1a. - Experimental ENDOR spectrum (solid curve) compared with computer simulation (dashed curve). Theoretical ENDOR spectrum due to $\mathrm{Br}^{79} b$ ) and $\mathrm{Br}^{81}(c)$. The numbers besides the dashed curves are the numbers $n_{\mathrm{s}}$ of equivalent nuclei which are responsible of that partion of curve. 
obtained is displayed versus $v_{\mathrm{E}}$ and $r_{\mathrm{hr}}$. Obviously it is not structured like the similar spectrum of $\mathrm{KI}$ [1] but it has enough peaks at $35,32.3,30.5$ and $28.5 \mathrm{MHz}$ to make possible a theoretical fitting.

We made a computer simulation of this spectrum based on the theoretical hyperfine frequencies (2) and with linewidths adjusted for a best fit. As expected the linewidths used here are larger than those previously used for KI [8]. Each normalized line was multiplied by an intensity factor

$$
n_{\mathrm{s}} l_{\mathrm{E}}^{2}\left\{1-\exp \left[-\left(v_{\mathrm{hf}} / \Delta\right)^{2}\right]\right\},
$$

$\Delta=5 \mathrm{MHz}$ to reflect the dependence on the number of equivalent nuclei, $n_{\mathrm{s}}$, in each shell, to reflect the fact that the probability for flipping a nuclear spin goes as $I_{E}^{2}$, and to reflect the fact that cross-relaxation effects tend to short-circuit the ENDOR at low value of $v_{n f}$. The best fit is shown in figure $1 a$, dashed curve. To obtain this fit we shifted pratically only one frequency clearly indicated in figure 2 , circles, where the

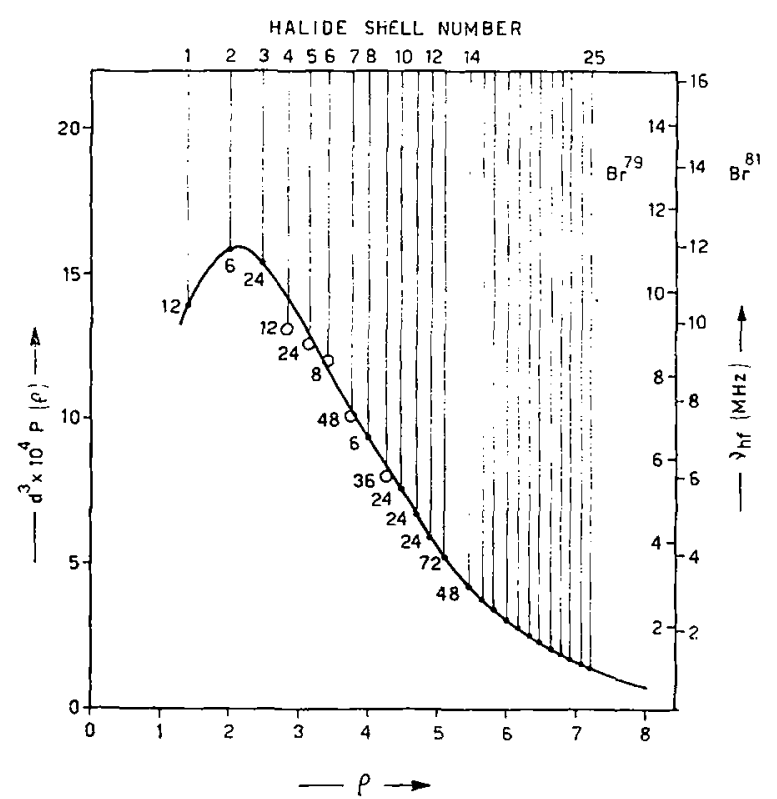

FIG. 2. - Probability density $P(\rho)$ versus units of the lattice parameter $d=3.298$. Solid curve is eq. (4) for " 0.48 . The dots and circles represent the frequencies used in the computer simulation. The number besides each dot or circle is the number $n_{\mathrm{s}}$ of equivalent nuclei of the halides shell.

hyperfine frequencies, dots, for $\mathrm{Br}^{71}$ and $\mathrm{Br}^{81}$ are reported. The solid line represents the function $P(p)$ and the number besides each dot or circle represents the number $n_{\mathrm{s}}$ of equivalent nuclei in cach shell. In figure $1 b$ and $1 c$ the separate spectra of ENDOR for $\mathrm{Br}^{79}$ and $\mathrm{Br}^{81}$ are given: the sum of the two gives the dashed line in figure $1 a$. The fit is obtained with $\eta=0.48$ and $A=2990$. values similar to the previous ones obtained using only the maximum hyperfine frequency. However these values are now more reliable and the uncertainty upon them is much smaller. For example a variation of 0.01 in the value of 17 makes the fitting almost impossible.
The RES radiative lifetime, $\tau=2 \times 10^{-6} \mathrm{~s}$, implies for the squared transition matrix element, $|x|^{2}$, a value between $0.0076 d^{2}$ and $0.0110 d^{2}$, the two limits obtained with and without local field correction. But $|x|^{2}$ is also given by :

$$
|x|^{2}=|<| \mathrm{s}|x| 2 \mathrm{p}>\left.\right|^{2}
$$

where $|2 p\rangle$ is the RES wavefunction, whose probability density is given by (4) and $\mid \mathrm{Is}>$ is the wavefunction for the non-relaxed ground state. We take the expression proposed by Fowler [9] for $\mathrm{F}$ center in $\mathrm{NaCl}$ :

$$
1 \mathrm{~s}>=\frac{\alpha^{3 / 2}}{7 \pi^{1 / 2}}(1+\alpha r) \mathrm{e}^{-\alpha r} \text {. }
$$

Using (6) and (4) in (5) we obtain :

$$
|x|^{2}=\frac{1}{3} \frac{32^{2} \alpha^{3} \eta^{5}(\eta+6 \alpha)^{2}}{7} d^{2} .
$$

In order to remain in the interval of $|x|^{2}$ implied by the lifetime $\tau_{1} x$ has to vary between 2.23 and 2.09 . These numbers are respectively $\sim 3 \%$ and $\sim 9 \%$ smaller than the value used by Fowler for $\mathrm{NaCl}$.

The aim of this work was to test the reliability of the model which assignes to the RES of $F$ center a very diffuse $2 \mathrm{p}$-like [9], [10] wavefunction, in contrast with the very compact one for the ground state. The model was used successfully to explain the ENDOR spectrum in $\mathrm{KI}$ [8] and now, applied to the more complicated case of $\mathrm{KBr}$. it gives a satisfactory agreement with the partially resolved ENDOR spectrum.

In conclusion we can say that at least for $\mathrm{KI}$ and $\mathrm{KBr}$ no doubts remain on the diffuse wave function of the RES of F center. (See note added.)

We would like to acknowledge the constant support given us by C. D. Jeffries, Professor at the Physics Department of the University of California, Berkeley, where the experimental part of this work was done. Also we are indebted with Alvaro Di Salvo for the useful assistence with the computer calculation.

Note added. - Following completion of this manuscript. W. B. Fowler pointed out to us that the $|2 s\rangle$ wavefunction we used in a previous paper [8], while trying various admixtures with the $\mid 2 p>$ wavefunction. is not the correct one. Indeed both the wavefunctions there had the same value of $\eta$, which is the parameter responsible for their radial extent. On the contrary the correct $\mid 2 s>$ state seems to be a very compact one. In this case the $|2 s\rangle$ and $|2 p\rangle$ radial probability densities are almost the same for $\rho>\sqrt{2}$. (We would like to stress that our experimental data cannot give any information on the wavefunction of the RES for values of $\rho$ smaller than $\sqrt{2}$, which is the distance of the nearest halide shell.)

Hence the conclusion drawn in [8], that the RES of the $F$ center in $K l$ is a pure $\mid 2 p>$ like state is incor- 
rect ; this is also true for the RES of $\mathrm{KBr}$, which this paper deals with. However the form (4) chosen for the RES remains a good approximation to the correct $|2 s\rangle$ state probability density in the region of interest.

In the light of this new evidence the calculation for the matrix element $|x|^{2}$ seems to remain quite good even if there is some doubt of the correctness of the form (6) of the $\mid 1 \mathrm{~s}>$ state, as pointed recently also by S. Wang et al., Phys. Rev. B 7 (1973) 1695. There is some evidence that the $|\mathrm{I} s\rangle$ state actually has a "long tail" that makes the matrix element much bigger than we once thought.

In conclusion we have determined the radial probability density of the RES with great accuracy and in our opinion this should be of considerable value to those who are trying to understand the relaxation process of the $F$ center. However our results do not bear directly on the question of $|2 s\rangle$ versus $|2 p\rangle$ state !

\section{References}

[1] Mollenauer, L. F., Pan, S. and Winnacker, A., Phy's. Rev. Lett. 26 (1971) 1643

[2] Mollenauer, L. F. and Pan, S., Phys. Rev. B 6 (1972) 772.

[3] Mollenauer, L. F., Pan, S. and Yngvesson, S., Phys. Rev. Lett. 23 (1969) 683.

[4] Abragam, A. and Bleaney, B., Electron Paramagnetic Resonance of Transition Ions (Clarendon, Oxford) 1970, 228.

[5] Fröhlich, D. and Mahr, H., Phys. Rev. 141 (1966) 692.

[6] Hoare, F. E., JaCkson, L. C. and KurTi, N., Experimental Cryophysics (London, Butterworths) 1961, 313.
[7] Honda, S. and Tomura, M., J. Phys. Soc. Japan 33 (1972) 1003.

[8] Mollenauer, L. F. and Baldacchini, G., Phys. Rev. Lett. 29 (1972) 465. In figures 1 and 2 of this work we made a mistake. The number of equivalent nuclei in the halide shells 7 and 13 are 48 and 72 respectively instead of 30 and 56 as stated there. This mistake reflects on the computer simulation, figure 2 , in the sense of a better agreement between theory and experiment with a new value for the cross-relaxation constant $\Delta=10.8 \mathrm{MHz}$.

[9] Fowler, W. B., Phys, Rev. 135 (1964) 1725.

[10] Bosi, L., Podini, P. and Spinolo, G., Phys. Rev. 175 (1968) 1133.

\section{DISCUSSION}

A. M. Stoneham. - a) Ham's most recent work indicates that the lowest relaxed excited state should be almost entirely s-like, and that any large p-admixture would be inconsistent with other work on the relaxed state;

b) Ham's analysis of the $\mathrm{KCl}$ F-centre suggests that s-p admixture is enough to explain the long lifetime, even without an extended excited state. It would be especially nice to look at this system by your methods.

G. Baldacchini. - a) I would like to add that
Ham feels that only an s-like wave-function could explain the isotropy of the ESR lines and ENDOR spectra. In any case at moment there is not a complete theoretical work on the nature of the RES.

b) Unfortunately 1 don't see now how to overcome the experimental difficulties arising from the short lifetime of the $\mathrm{F}$-center in $\mathrm{KCl}$. Even more the $g$ factors of the ground state and the RES are not too different from each other and there would be an overlapping of the two EPR lines which is really disturbing in our method. 